J. Neurol. Neurosurg. Psychiat., 1954, 17, 276.

\title{
PATHOLOGICAL FINDINGS IN TEMPORAL LOBE EPILEPSY
}

BY

\author{
ALFRED MEYER, MURRAY A. FALCONER, and ELISABETH BECK \\ From the Department of Neuropathology, Institute of Psychiatry, and the Guy's-Maudsley \\ Neurosurgical Unit, Maudsley Hospital, London
}

Temporal lobe epilepsy, similarly to focal epilepsy arising elsewhere in the brain, can result from a wide variety of lesions that may involve the temporal lobe. Some of these may be of sufficient size, as for example most tumours, cirsoid angiomas, porencephalic cysts, and the scars of penetrating head injuries, so that their presence can usually be revealed by the appropriate clinical and radiological examinations. More often, however, the neurological examination is negative (apart from electroencephalography) and cerebral arteriography and encephalography do not disclose any such tangible abnormality. The underlying pathological basis then is a matter for speculation and conjecture. For more than a century it has been known that sclerosis of Ammon's horn (hippocampus) is a finding frequently observed at necropsy in the brains of chronic epileptics. It was noted by Spielmeyer (1927) in $80 \%$ of 126 epileptic brains, but until recently no attempt had been made to relate this particular lesion to the manifestations of temporal lobe epilepsy.

Our interest in this subject was specially aroused by the paper of Penfield and his collaborators (Earle, Baldwin, and Penfield, 1953) claiming that " incisural sclerosis" was the most common cause of temporal lobe epilepsy. It had been found by them in 100 out of 157 patients $(63 \%)$ submitted to operation. The extent of this sclerosis ranged from involvement of a single gyrus to involvement of the entire temporal lobe, but the mesial and inferior portions of the lobe (uncus, hippocampal gyrus) and first temporal gyrus were the areas most frequently involved. However, they did not specify whether the sclerosis of the hippocampal gyrus also extended to Ammon's horn. They further claimed that this incisural sclerosis is caused exclusively by herniation of the temporal lobe into the tentorial opening during the head moulding of parturition. This herniation, they held, occurs at a time when the temporal lobe is poorly developed and more susceptible to damage through compression of the anterior choroidal, posterior cerebral, - and middle cerebral arteries against the tentorial edge with consequent local anoxia. This is a challenging hypothesis that runs counter to the widely accepted views that sclerosis of Ammon's horn, when it occurs in epileptics, is secondary to severe major epileptic seizures. In their remaining 57 cases Penfield and his associates found a post-natal pathology, usually in the form of tumours or the sequelae of head injury or infection.

\section{Case Material}

We have therefore analysed the biopsy findings in 18 cases of temporal lobe epilepsy treated by temporal lobectomy in the Guy's-Maudsley Neurosurgical Unit between May, 1951, and July, 1953. Each case was one of intractable epilepsy, usually with a range of seizures of minor, amnesic, and grand mal types (Falconer, 1954), in which the electroencephalographic studies had disclosed an epileptic focus confined to or largely predominant in one temporal lobe, while the clinical and neuroradiological examinations had not revealed a tangible structural lesion. Many of the patients suffered also from an associated personality and behaviour disorder. The clinical, operative, and electroencephalographic data in the first 14 of these cases have already been reviewed by Hill, Falconer, and Pampiglione (1953). Several other cases of temporal lobe epilepsy due to large structural lesions, such as readily recognizable tumours, porencephalic cysts, and angiomas, were also operated on during the same period, but these were excluded as the operative intervention in them was limited to an excision of the lesion, whereas the cases in the present series were treated by an excision of the greater part of the temporal lobe.

Most of our biopsy specimens contained the anterior half to two-thirds of the temporal lobe, usually from the middle temporal convolution laterally to the hippocampal gyrus medially. The first temporal convolution, if it was included at all, was usually incomplete ; the Ammon's horn was occasionally missing, particularly in the earlier 
specimens. Whenever there was no macroscopically obvious focal abnormality, the blocks into which the specimen was divided were embedded in celloidin and cut in serial sections. Usually only a small amount of tissue was left for the techniques applicable on frozen sections.

Histological investigations of biopsy specimens have their special difficulties. Nerve cells and fibres almost invariably show artefacts. Extravasations of blood and mechanical damage during the operation obscure the architecture. Moreover, some staining reactions, and in particular the glial fibre stains so important for the assessment of age and severity of a lesion, are seldom satisfactory. In this regard Mallory's phosphotungstic-acid-haematoxylin stain proved to be superior to Holzer's stain. We had to bear these difficulties constantly in mind in the interpretation of the lesions.

\section{Histological Findings}

Our case material on analysis proved to be divisible into two groups corresponding to those of the Montreal workers. The first group comprises four cases with small but macroscopically clear-cut circumscribed lesions. The second and larger group consists of cases in which such local lesions were absent, and in which the changes were essentially of a more diffuse and microscopic character.

Group 1 : Small, Macroscopic, Focal Lesions.In three instances these proved to be small tumours, while the fourth case was one of traumatic cicatrix. One of the tumours was a small meningioma situated on the inferior surface a little behind the pole and extending for about $2 \mathrm{~cm}$. within the fusiform gyrus (Fig. 1). The histological diagnosis: in this case was a surprise because there had been an antecedent head injury, and at operation the case was considered one of traumatic meningo-cortical cicatrix. The two other tumour càses' havaalready been reported by Falconer, Pond, Meyer, and Woolf (1953) and would come under the generic term of hamartomas. In these a small calcified lesion similar to what Penfield and Ward (1948) described as a "haemangioma calcificans" was found, in one instance occupying the temporal lobe inferior to the temporal horn, and in the other the uncus and parts of the amygdaloid nucleus which are entirely obscured (Fig. 2). In the latter case the lesion was not noted at operation, and indeed its presence was not realized until the sections came to be examined. The well defined localization of the lesion in this case much resembles that in Hughlings Jackson's original case although the lesion there was a vascular one.

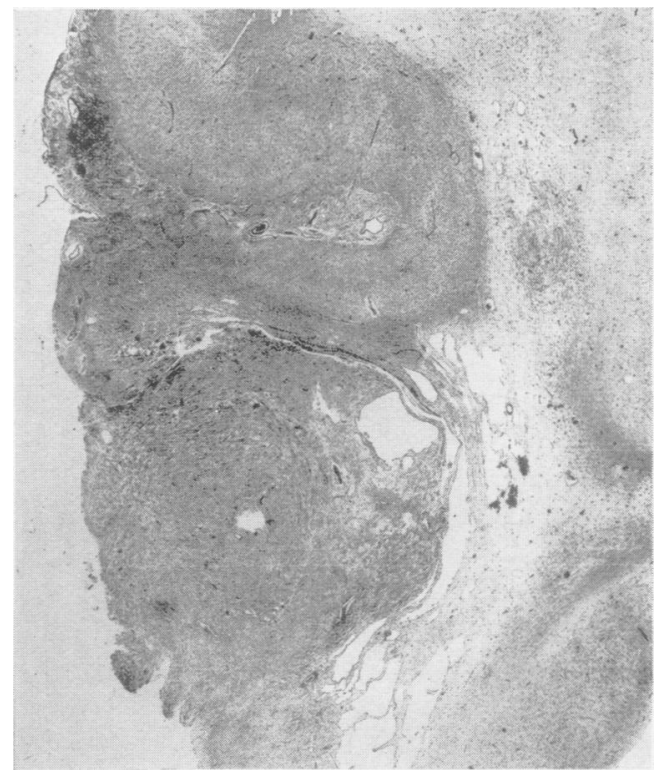

FIG. 1.-Small, well encapsulated meningioma indenting the fusiform gyrus. Van Gieson stain. $\times 5 \cdot 5$.

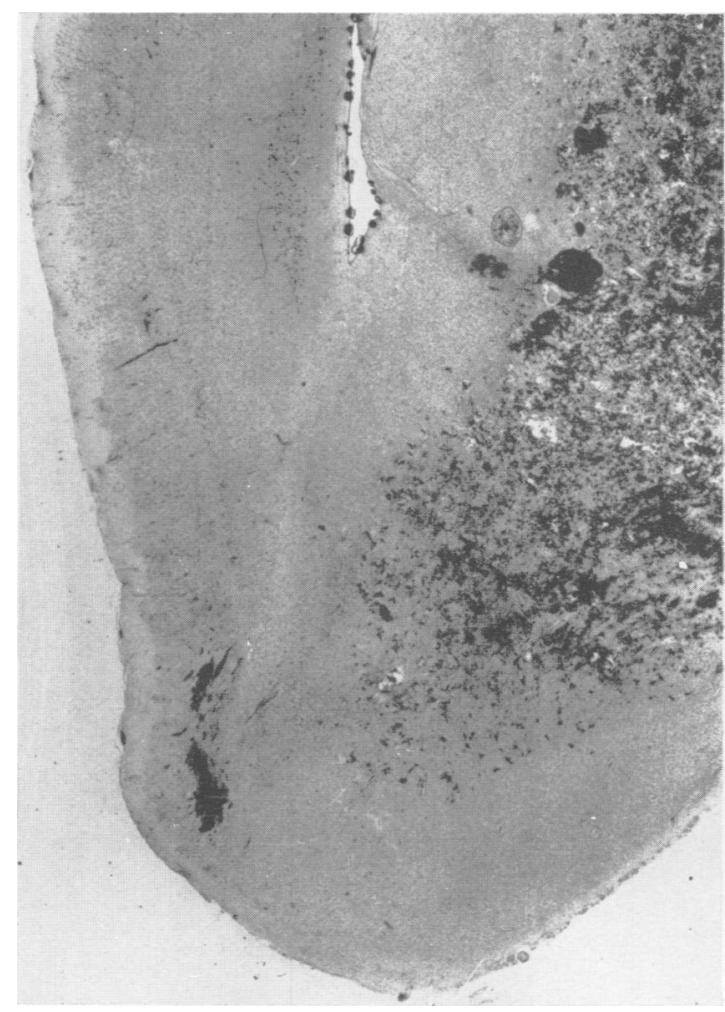

FIG. 2.-Haemangioma calcificans situated within the region of the uncus. Haematoxylin and eosin stain. $\times 8$. 
Group 2 : Non-circumscribed Microscopic Lesions. -This group contains 14 cases, and the relevant histological findings are set out in Table I under the headings of meningeal fibrosis, marginal gliosis, cortical atrophy, white matter gliosis, and Ammon's horn sclerosis. It will be seen that in each case some abnormality was noted although in greatly varying proportions. It can also be noted that frequently the skull on the side of the resected lobe was smaller than on the opposite side, suggesting a disturbance of development dating from early life, and that even more frequently the cerebral hemisphere on the operated side was small or showed other evidence of atrophy.

Meningeal Fibrosis.-Meningeal fibrosis was particularly marked in Cases 4, 5, and 6. The meningeal affection in these cases may possibly indicate trauma (neonatal or post-natal) or infection. There were some scanty lymphocytic and leucocytic infiltrations in the meninges of Cases 4 and 5, but their aetiological significance is doubtful in view of the long history in both cases.

FIG. 3.-Case 6 : marginal gliosis. Mallory's phosphotungstic acidhaematoxylin stain. $\times 300$.

Fig. 4a.-Case 2: cortical atrophy and white matter sclerosis. Nissl stain. $\times 3$.

Fig. 4b.-Normal temporal lobe for comparison. Nissl stain. $\times 3$.

Fig. 4c.-Case 2 : severe laminary nerve cell loss within the third layer. Dense gliosis of the white matter. Nissl stain. $\times 25$.

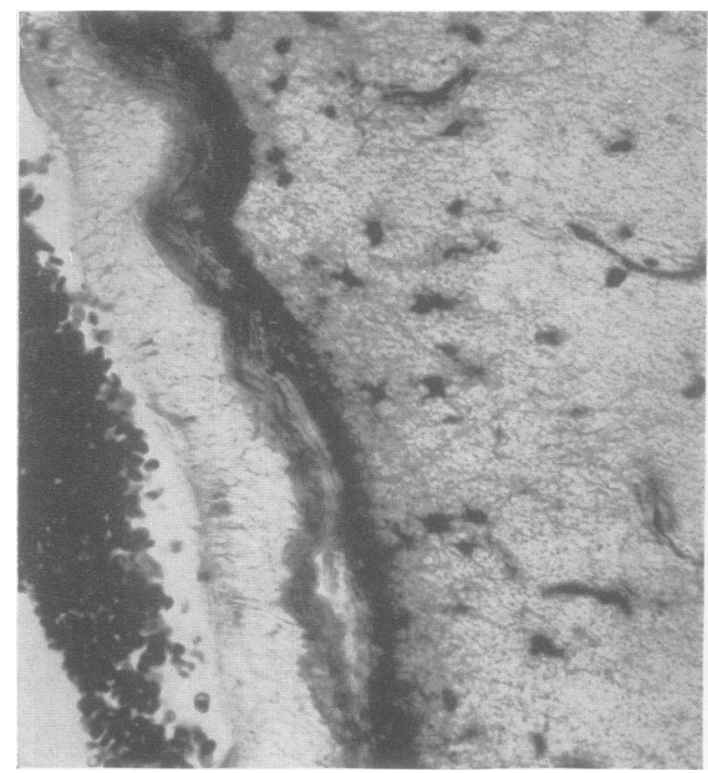

Fig. 3

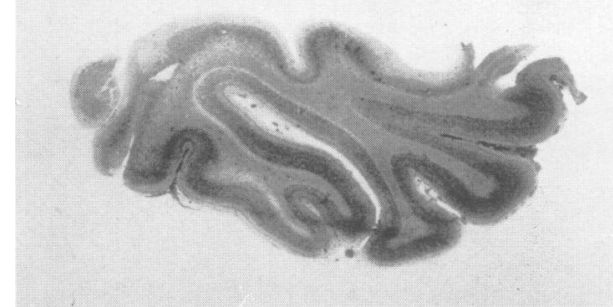

Fig. 4a

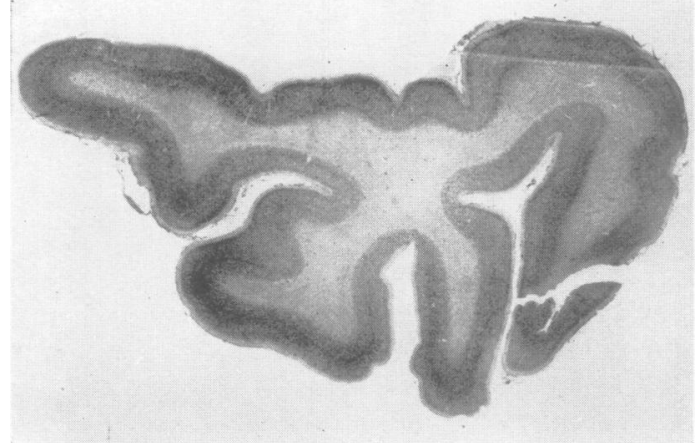

Fig. 4b

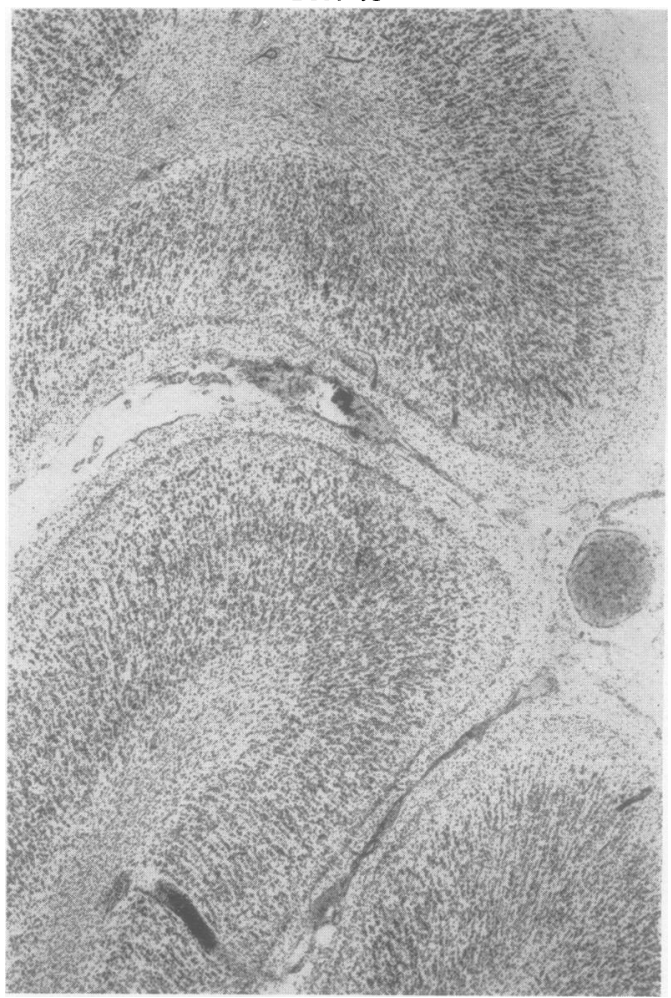

Fig. 4c 
Marginal Gliosis.-Marginal gliosis of widely varying degree and extent was seen in every case. It was usually demonstrated fairly satisfactorily by Mallory's phosphotungstic-acid-haematoxylin stain as Fig. 3 indicates.

Cortical Atrophy.-Cortical atrophy was in several cases severe and generalized. Fig. 4a shows its degree in the anterior part of the temporal region near the pole in Case 2 in comparison with the corresponding region in a normal brain (Fig. 4b). A higher magnification (Fig. 4c) shows a generally thin cortex with the neuronal loss and glial overgrowth being particularly marked in layer 3 and, less often, in layer 2. This illustration compares well with figures 64 and 65 of Scholz (1951) in which he demonstrates laminary atrophy in postepileptic lesions. Quite often the laminary atrophy was more localized and was found with particular frequency in the depth of the sulci, e.g., around the collateral sulcus (Fig. 5).

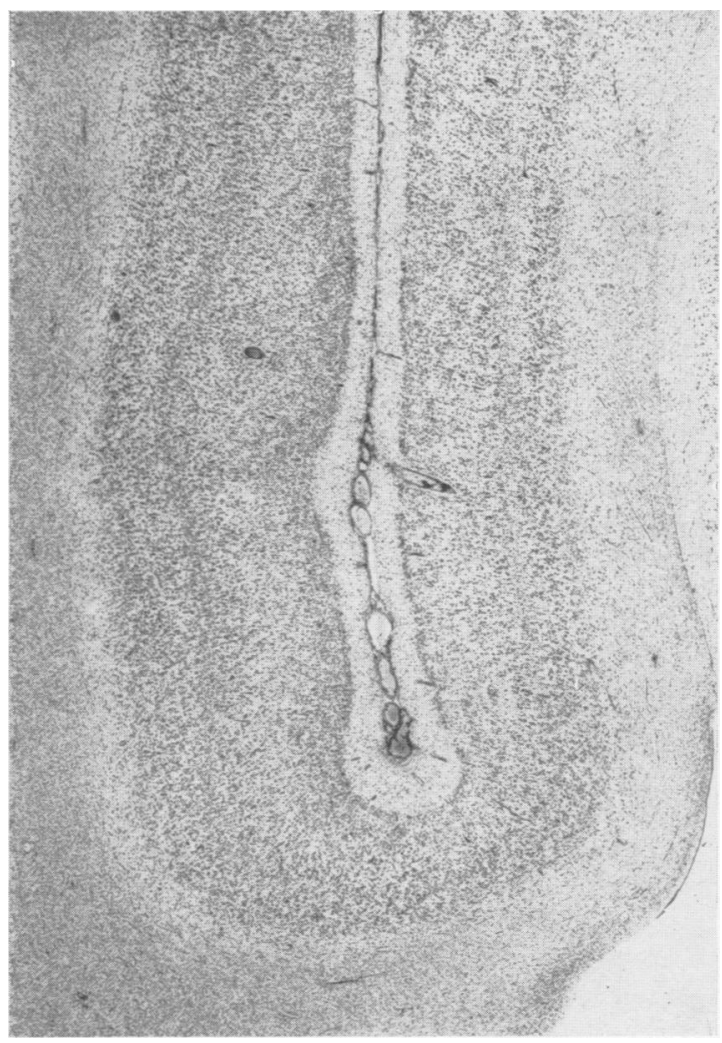

Fig. 5.-Case 14 : laminary atrophy of the third layer around the collateral sulcus and dense gliosis of the white matter. Nissl stain. $\times 13$.

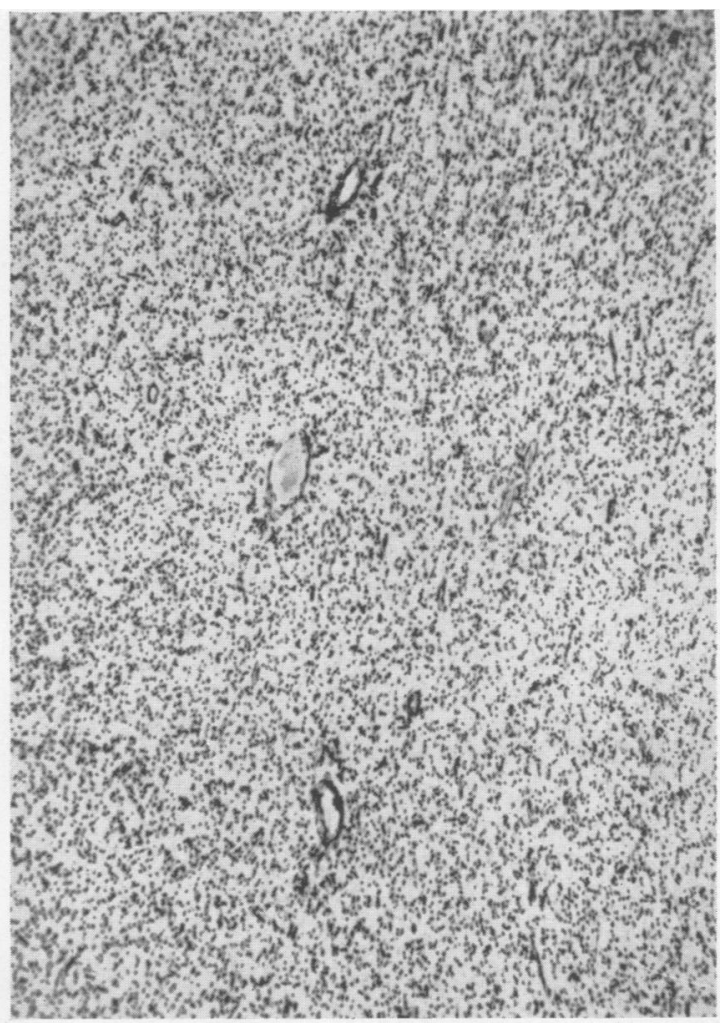

FIG. 6.-Case 10 : severe gliosis in the white matter of the hippocampal gyrus. Nissl stain. $\times 68$.

Glial Sclerosis of White Matter.-Glial sclerosis of the white matter often accompanied the cortical atrophy, but in at least two cases it was more marked than the changes in the cortex. It was usually densest in the vicinity of the temporal horn and in the hippocampal gyrus (Figs. 5 and 6). Owing to the failure of glial fibre stains conclusions had to be drawn from the number of oligodendroglial cells and astrocytes in Nissl-stained slides. In none of the 14 cases of this group was there any appreciable demyelination, a fact of some interest since it renders a measles encephalitis, which as a rule is of the demyelinizing post-vaccinal type, unlikely as a cause.

Sclerosis of Ammon's Horn.-Fig. 7 illustrates the sclerosis of Ammon's horn, as found in Cases 10 and 3, compared with a normal hippocampus. In Case 10 (Figs. 7b and c) there was sclerosis of the Sommer sector (hl) and endplate (h3-h5) which also showed well after Holzer staining. (This patient died and these sections were taken after death from the unoperated hemisphere ; there was 


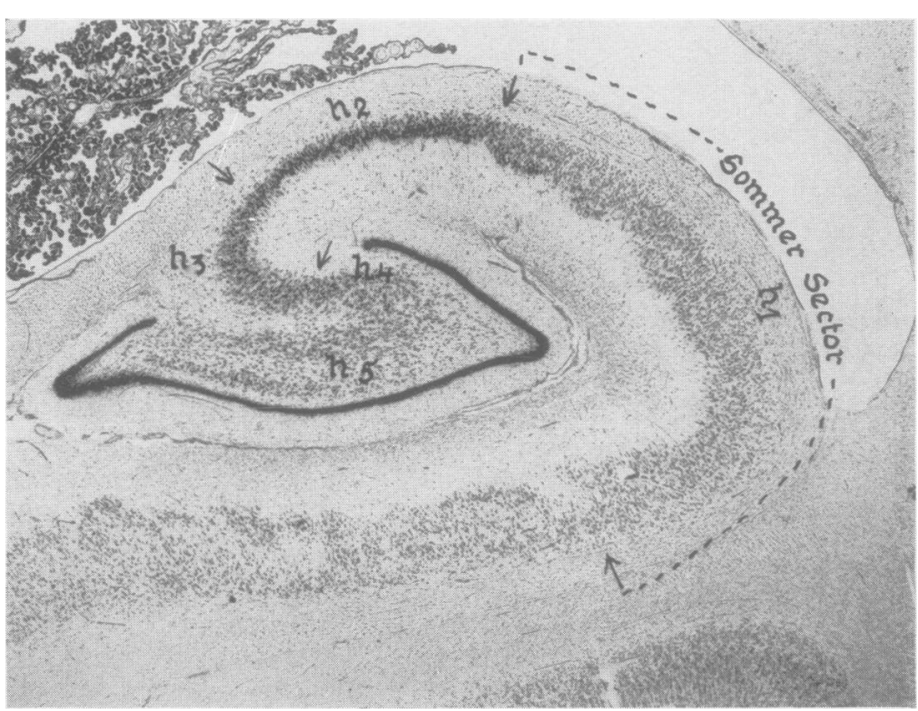

Fig. 7b
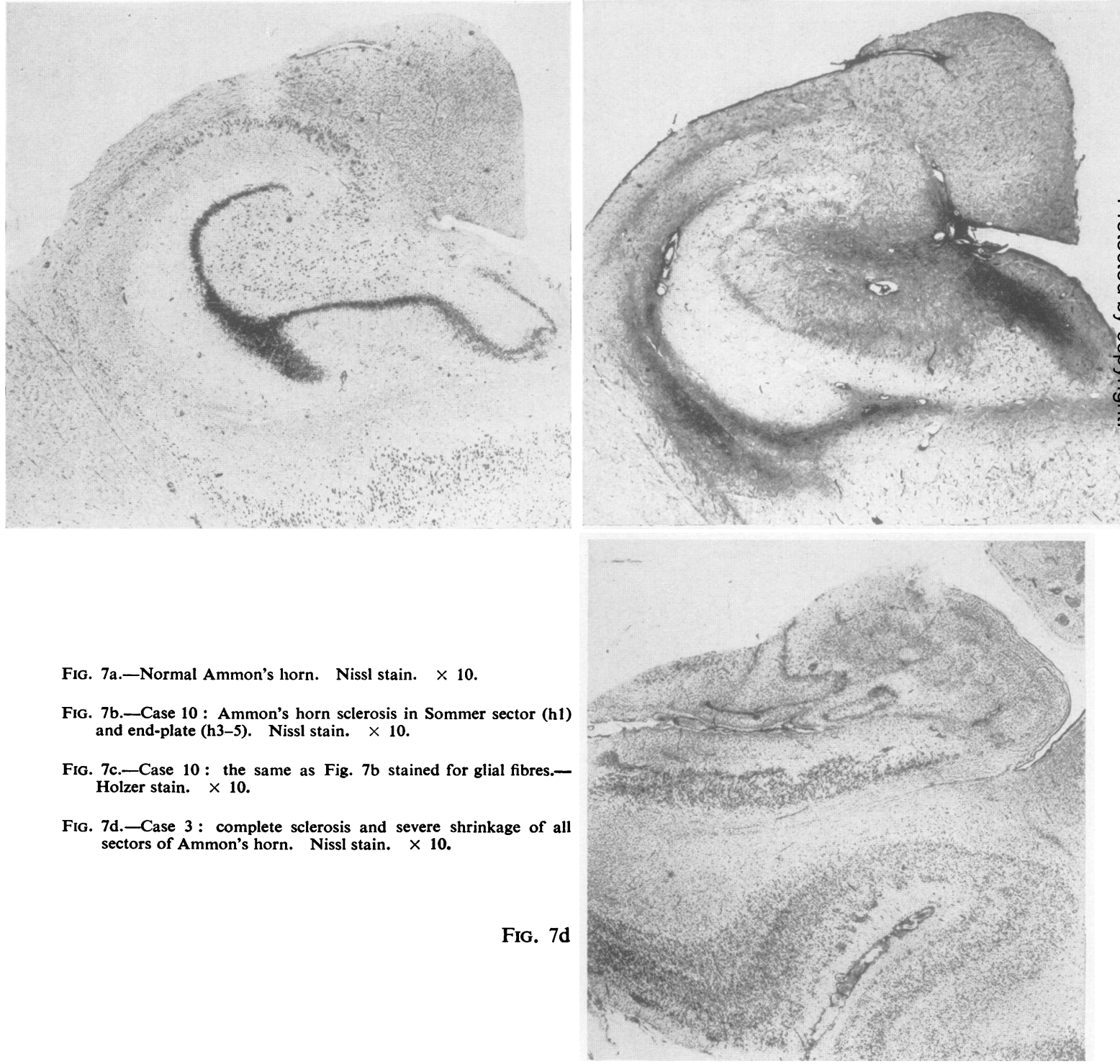

Fig. 7a.-Normal Ammon's horn. Nissl stain. $\times 10$.

FIG. 7b.-Case 10 : Ammon's horn sclerosis in Sommer sector (h1) and end-plate (h3-5). Nissl stain. $\times 10$.

Fig. 7c.-Case 10 : the same as Fig. $7 \mathrm{~b}$ stained for glial fibres.Holzer stain. $\times 10$.

Fig. 7d.-Case 3: complete sclerosis and severe shrinkage of all sectors of Ammon's horn. Nissl stain. $\times 10$.

FIG. 7d 
no sclerosis of Ammon's horn in the biopsy specimen.) Relatively little shrinkage was present compared with the much severer shrinkage in Case 3 (Fig. 7d). In Case 10 the sclerosis of Ammon's horn was not associated with cortical atrophy, whereas in Case 3 it was part of a severe atrophy of all available temporal convolutions.

Table I gives the exact extent of the hippocampal lesion in the 12 cases in which this could be determined. It will be seen that a definite Ammon's horn sclerosis was present in seven cases although to a varying degree. In four further cases, although there was no appreciable loss of nerve cells in any sector, the glial cells were definitely increased in number. We have recorded this latter change as of uncertain significance ( $(-)$. In the remaining case (Case 13) excessive siderosis (pseudocalcium) was observed in blood vessel walls. In two cases ( 5 and 6 ) no tissue of Ammon's horn was available for investigation.

\section{Discussion}

Some pathological change was thus observed in every case, while the post-operative follow-up studies at periods ranging from one to three years show that most cases have been strikingly benefited by removal of the diseased temporal lobe. The degree of improvement was particularly striking in those cases in which sclerosis of Ammon's horn was found. In only three instances was the therapeutic result not definitely beneficial. One patient, Case 10, died eight days after operation. Two others, Cases 9 and 14, were only slightly improved as regards the frequency and severity of their fits, and not at all as regards their personalities. In both of these there was evidence of involvement of the remaining temporal lobe as well. In Case 9 the pre-operative radiographs showed that the hemicranium and cerebral hemisphere on the unoperated side were smaller than on the operated side, while postoperatively the E.E.G.s have shown a pronounced epileptic focus on the unoperated side. Again in Case 14 the pre-operative radiographs gave evidence of a bilateral disturbance, as did the E.E.G.s.

The fact that following temporal lobectomy the clinical features were so frequently improved strongly suggests that the pathological changes were

TABLE I

EXTENT AND DEGREE OF SEVERITY OF AMMON'S HORN SCLEROSIS

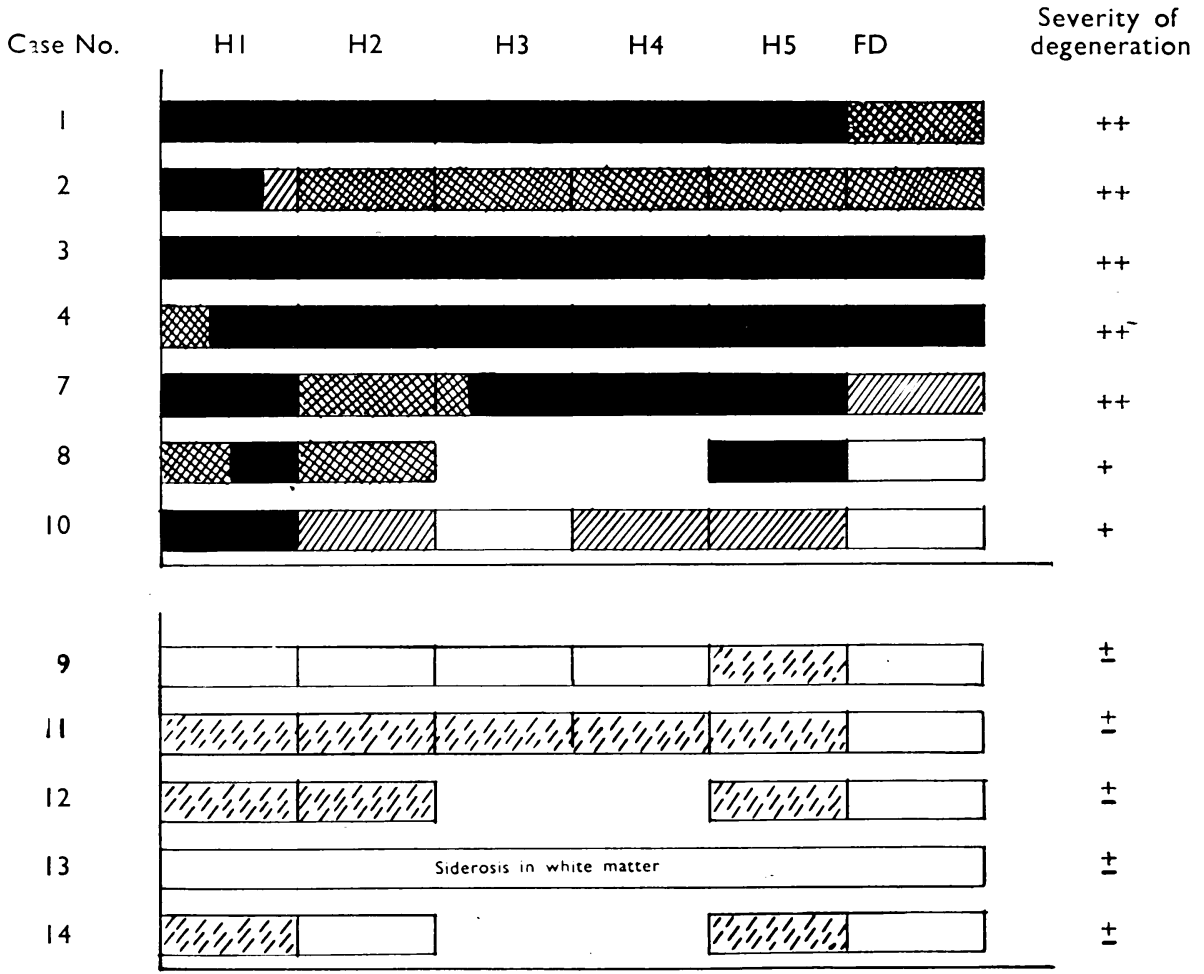

H1-5 correspond to Rose's subdivision of the Ammon's horn, H1 being approximately the Sommer sector. FD = fascia dentate.

Solid black indicates complete degeneration; cross hatching, single, and broken lines gradual decrease in severity. 
somehow responsible for the epilepsy, which had improved as a consequence of their removal. This is in keeping with the general clinical observation that the mechanisms responsible for epilepsy are dependent not so much on the nature of the pathological change as on its site.

Several points of importance emerge from the presence of Ammon's horn sclerosis in our material. Its incidence (if we leave out uncertain cases) is no higher than has been reported in overall statistics of epileptic brains (Spielmeyer, 1927; Scholz, 1951 ; Sano and Malamud, 1953). Sano and Malamud found Ammon's horn sclerosis in 29 out of 50 anatomically investigated cases. In those cases suitable for such analysis they report a significant correlation between the presence of Ammon's horn sclerosis and the occurrence of clinical and electroencephalographic features of temporal lobe epilepsy during life. From these findings one might have expected a higher incidence of Ammon's horn sclerosis in our material than seven cases out of 12 . In all our cases with typical Ammon's horn sclerosis the onset of temporal or generalized epilepsy was below the age of 10 years. Although it is established, of course, that Ammon's horn sclerosis identical in appearance and degree may be produced in epilepsy of onset at a later age it would be of great interest if the frequency of its incidence were higher in infancy and childhood. We are just completing an anatomical analysis of 17 patients who became epileptic after leucotomy (to be published separately), and in only one of these an Ammon's horn sclerosis was found.

The Ammon's horn sclerosis found in temporal lobe epilepsy is in every detail identical with that occurring generally in epileptic brains.

The laminary loss of neurons in the third layer, particularly in the depth of the sulci, together with the typical sclerosis of the Ammon's horn, speaks in favour of anoxia as the causal mechanism in many cases of our second group. Penfield and his collaborators assumed that this anoxia occurred at birth. Against this must be set the widely accepted view elaborated by Spielmeyer and his school, particularly by Scholz $(1951,1953)$, that Ammon's horn sclerosis in epilepsy results from the functional vascular and anoxic disturbances which accompany (or perhaps precede) severe generalized convulsions. These authors have provided convincing evidence that in some areas the early stages of ischaemic necrosis in the vulnerable sector could not have been produced otherwise than by a preceding severe generalized convulsion. Scholz claims that such convulsions may be particularly deleterious in early infancy and childhood, especially if they occur in connexion with the exanthematous fevers, whooping cough, acute gastric infections, and acute allergic conditions. The damage in the brain thus caused may be considerable, and may reach the proportions of total, lobar, or hemisclerosis and may also affect the cerebellum, thalamus, and other subcortical centres. Most lesions described in our second group would theoretically fit into the Scholz category of post-epileptic damage. Of course, Scholz would not deny that anoxia occurring without convulsions, including, for example, anoxia at birth, may have a similar effect on the brain.

That birth injury is only one possible aetiological factor is well illustrated by a post-mortem observation (outside our operative series). It concerned a boy with no history of birth difficulty who at the age of 8 developed Still's syndrome with swelling of joints, transient rashes, and fever. In the course of this illness a series of generalized convulsions occurred suddenly, lasting for five hours. He remained comatose for three days and developed pneumonia. On recovering from pneumonia he was found to be demented. On several occasions he had recurrences of status epilepticus, but he also had a variety of minor fits including screaming attacks and sudden autonomic disturbances which were thought to point to the temporal lobe. He died 12 months after the first epileptic status. The brain showed widespread damage which will be described in detail in a subsequent paper (Meyer, Beck, and Shepherd). The temporal lobe was severely affected, and, as Fig. 8 demonstrates, showed general cortical atrophy which was particularly marked in the first temporal convolution (and the whole region surrounding the Sylvian fissure) and in the medial convolutions including the Ammon's horn. All the lesions had the characteristics of relatively recent sequelae of anoxia and none of them could histologically have been older than the first status epilepticus. This case thus shows conclusively that the same combination of lesions of the hippocampal region and the first temporal convolution which Penfield and his colleagues ascribed exclusively to a specific mechanism of anoxia and herniation at birth may occur in later life, in this instance, presumably, as a sequel to serial epileptic convulsions at the age of 8 years. It may be mentioned that particular attention was directed in this case to organic disease of the blood vessels but none was found.

The complexity of the aetiological interpretation is reflected in Table II in which some relevant clinical data are plotted against the pathological changes in the 14 cases of our second group. Pathological birth was reported in six cases, but in 


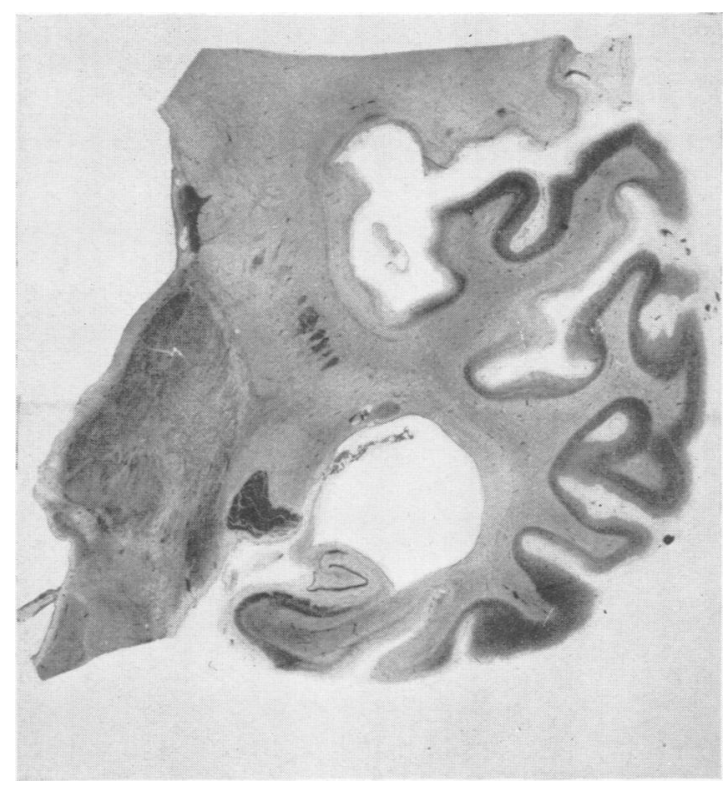

Fig. 8.-Case H. (post-mortem specimen). Atrophy of the tempora lobe and Sylvian region. Within the temporal lobe the atrophy is most marked in the first temporal convolution and the most medial convolutions including the Ammon's horn. The latter shows degeneration of nerve cells within the Sommer sector and end-plate. Nissl stain. $\times \mathbf{1 . 6}$.

two (Cases 2 and 9) the onset of symptoms was not before the second decade which renders any aetiological connexion tenuous. There is no conspicuous correlation of a history of difficult birth with the major degrees of damage to the Ammon's horn. In Cases 1 and 7, for instance, there was severe Ammon's horn sclerosis without a history of birth injury, while in Cases 9 and 12 with definite birth trauma there were only minor Ammon's horn changes. The onset of epilepsy coincided with childhood fevers in three cases; in one of these (Case 1) an infantile hemiplegia suddenly appeared as a complication of measles, possibly due to a vascular accident (arterial or venous thrombosis) or to an encephalitis ; in the other two (Cases 7 and 8 ) the epilepsy was ushered in by severe serial generalized convulsions. Only in Case 14 was a definite post-natal head injury recorded.

Major convulsive seizures were reported in eight cases and are apparently excluded in three (Cases 4, 11 , and 12). In the remaining cases no such seizures were described, but our information covering all phases of each patient is not sufficiently comprehensive to be certain. Only in Cases 7, 8, and 14 is it definitely known that severe generalized convulsions preceded the characteristic temporal lobe attacks.
On the other hand, a typical Ammon's horn sclerosis was found in three cases in which the occurrence of major attacks was, as far as one can tell, either excluded or uncertain. Of these, Case 3 had a history of severe birth injury and subsequently exhibited a congenital type of nystagmus. Case 4 also had a history of severe birth injury, while in Case 8 both birth injury and chickenpox are recorded.

Final conclusions, obviously, must wait until a larger number of cases and more detailed information on the clinical and electrophysiological aspects will be available. We are also inevitably handicapped by not knowing whether similar changes are found in the unoperated temporal lobe or in other parts of the brain. It already emerges, however, from the analysis of the present series that birth injury as a cause of temporal lobe epilepsy in the absence of convulsions is proved or likely in at most only three cases $(3,4$, and 12), and that a history of birth injury does not correlate consistently with the presence of Ammon's horn sclerosis. On the other hand, it seems fairly certain that in Cases 1,7 , and 8 events complicating measles, "teething", and chickenpox respectively were responsible for the onset of temporal lobe epilepsy. To these three cases must be added the post-mortem case in which the combination of lesions in the first temporal convolution and Ammon's horn followed a severe status epilepticus during Still's syndrome at the age of 8 years.

It follows then that the aetiological concept of incisural sclerosis caused by tentorial herniation and anoxia at birth put forward by Penfield and his collaborators may have been an over-simplification, and that later events in infancy, childhood, and early adolescence play at least an equal, if not greater, part. This is in agreement with the views expressed by Gastaut (1953) in his recent critical review. Gastaut attached particular importance to trauma and encephalitis, especially measles encephalitis, but in our opinion encephalitis may not be as important as vascular accidents and other -anoxic conditions, and, in particular, the anoxia following serial epileptic convulsions during the fevers of childhood, acute gastric infections, and acute allergic conditions of this age period.

However, although their hypothesis requires some modification, the Montreal workers have done a great service by renewing attention upon the Ammon's horn sclerosis whose pathogenic and functional significance still remains an enigma. It is not intended to discuss this complex problem in this paper. Much interesting information on the functions of Ammon's horn has emerged from 
TABLE II

CORRELATION OF BIOPSY FINDINGS WITH CLINICAL DATA (EXCLUDING CASES OF TUMOUR) IN 14 CASES OF TEMPORAL LOBE EPILEPSY*

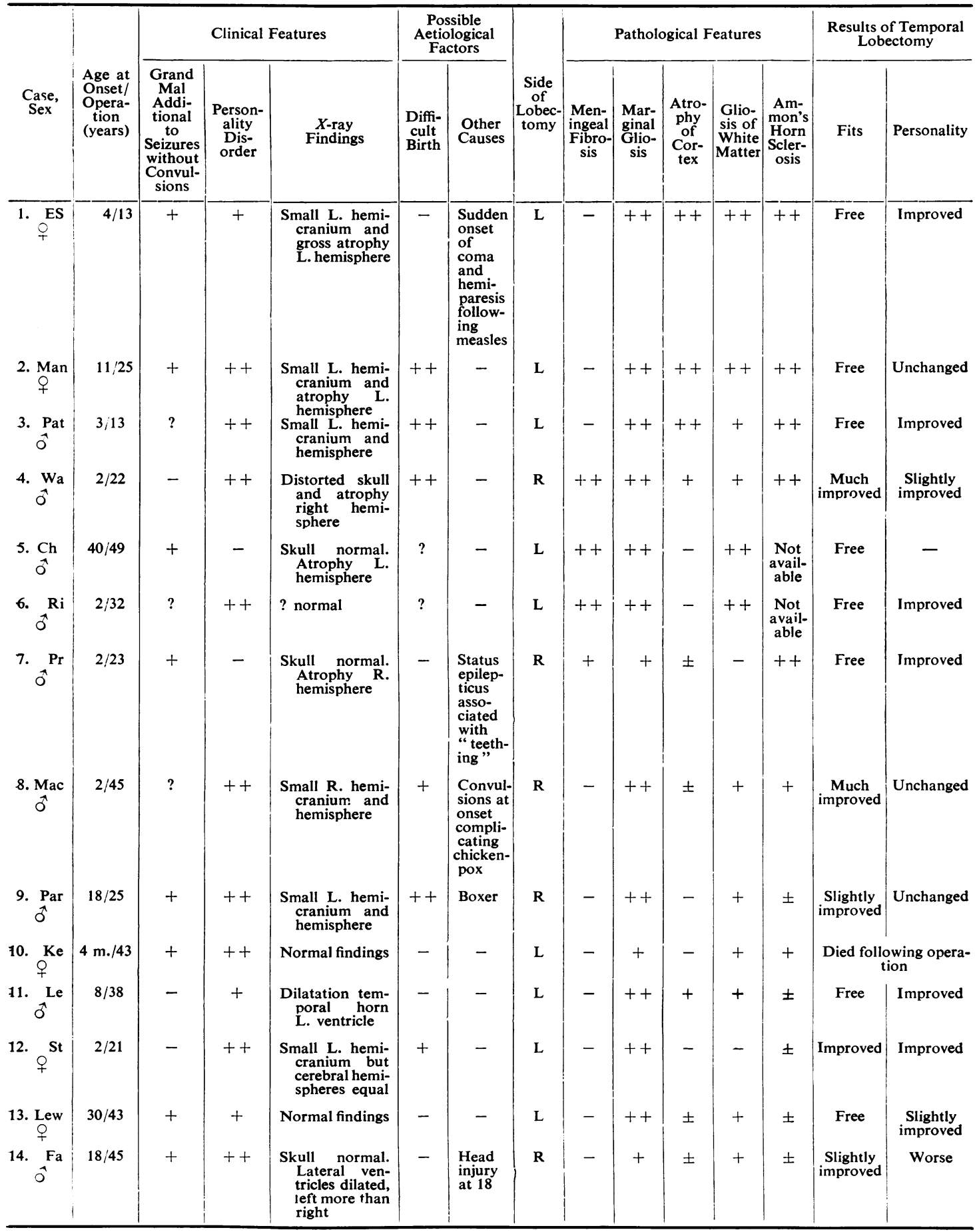

*Order approximately according to severity of the lesions. 
recent experimental physiological research carried out by McLean, Pribram, Kaada, and Delgado, and, from a different angle, by Gastaut and his associates (vide Gastaut, 1953). This structure is now regarded as forming part of the visceral brain or limbic system together with the uncus and amygdaloid nucleus and the so-called transitional cortex of the anterior insula, posterior orbital, and anterior cingular regions. Electro-anatomical investigations have demonstrated the close inter-relationship of these centres and their connexions with the diencephalon. Gastaut and others have shown in experimental animals that behavioural changes similar to those seen in temporal lobe epilepsy can be elicited from these areas by electrical or chemical stimulation. MacLean (1954) believes that the frontotemporal portion of the visceral brain is specifically concerned with the memories, feelings, and actions related to the oral senses, i.e., to functions which would obviously suggest its major importance to temporal lobe seizures. Other workers relate the visceral brain rather to more general functions. Papez, in his now famous paper, in 1937 regarded a similar combination of centres as a cerebral mechanism for the elaboration of emotion and emotional expression. Herrick (1933), Grünthal (1947), and Le Gros Clark (1950) saw in the Ammon's horn an unspecific activator of cortical function. Our knowledge in this field is still in a state of considerable flux, so that a certain reserve is indicated when basing hypothetical considerations upon it.

The next step in the investigations which our group are undertaking will be to coordinate clinical symptoms, electrocorticographic observations, and the effects of stimulation during the operation with the subsequent anatomical and pathological analysis. The importance of systematic post-mortem studies of clinically and electrophysiologically well-investigated cases of temporal lobe epilepsy must also be stressed. They are bound to give a more comprehensive and clearer picture of the histological changes than the investigation of isolated biopsy specimens, so difficult to interpret, could ever provide.

\section{Summary}

The pathological findings in 18 resected temporal lobes of patients suffering from temporal lobe epilepsy and one post-mortem brain have been reported. The surgical cases were all ones in which the pre-operative clinical and radiological studies had failed to demonstrate the presence of spaceoccupying or other gross circumscribed lesions, but in which electroencephalography had disclosed a unilateral epileptic focus.

The material can be divided into two groups. The first group of four cases contains three small tumours and one case of gross post-traumatic scarring.

The second group of 14 operative and one postmortem case is much larger and has been especially analysed. All except three of the operated cases were strikingly benefited by the removal of the temporal lobe. Lesions were found in all cases but varied considerably in quality and degree. In many cases a laminary atrophy, particularly of the third cortical layer, often situated in the depth of the sulci, was found and interpreted as a consequence of anoxia. Ammon's horn sclerosis was found in seven of 12 cases, interestingly in all cases with onset of the disease before the age of 10 . It was correlated neither with a history of birth injury nor with that of preceding major convulsions. Birth injury as the cause of the epilepsy was proved or likely in at most three cases. On the other hand, three cases of temporal lobe epilepsy followed a cerebral complication (probably vascular accident or encephalitis) during measles, status epilepticus following " teething", and chickenpox respectively. To these must be added a postmortem case in which lesions with characteristic distribution followed status epilepticus in the course of Still's syndrome at the age of 8 years.

It was concluded from this preliminary investigation that incisural sclerosis resulting from birth mechanisms cannot be maintained as an exclusive aetiology and that later events in infancy, childhood, and early adolescence play at least an equal part.

Our thanks are due to Dr. Denis Hill and Dr. D. A. Pond for investigating and referring for operation most of the patients studied in this series, to Dr. R. Hoare for the radiological examinations, and to Dr. W. Mitchell and Dr. G. Pampiglione for some of the clinical data. We are also indebted to Miss Edna Walker for her technical help and to the Bethlem Royal and Maudsley Hospital (Research Fund) for an expenses grant. Much of the information collected in this paper was communicated by one of us (A. M.) to the Association of British Neurologists at their spring meeting on April 23.

\section{REFERENCES}

Clark, W. Le Gros (1950). In Perspectives in Neuropsychiatry, pp. 9-24. Edited by D. Richter. H. K. Lewis, London.

Earle, K. M., Baldwin, M., and Penfield, W. (1953). Arch. Neurol. Psychiat., Chicago, 69, 27.

Falconer, M. A. (1954). Brit. med. J., 2, 939.

- . Pond, D. A., Meyer, A., and Woolf, A. L. (1953). Journal of Neurology, Neurosurgery and Psychiatry, 16, 234.

Gastaut, H. (1953). Epilepsia, 2, 29.

Grünthal, E. (1947). Mschr. Psychiat. Neurol., 113, 1.

Herrick, C. J. (1933). Proc. nat. Acad. Sci., Washington, 19, 7.

Hill, D., Falconer, M. A., and Pampiglione, G. (1953).' Proc. roy. Soc. Med., 46, 965 .

MacLean, P. D. (1954). J. Neurosurg., 11, 29.

Meyer, A., Beck, E., and Shepherd, M. Journal of Neurology, Neurosurgery and Psvchiatry. To be published.

Papez, J. W. (1937). Arch. Neurol. Psychiat., Chicago, 38, 725.

Papez, J. W. (1937). Arch. Neurol. Psychiat., Chic

Penfield, W., and Ward A. (1948). Ibid., 60, 20.

Sano, K.̈̈and Malamud, N. (1953). Ibid., 70, 40.
Scholz, W. (1951). Die Krampfschädigungen des Gehirns. Springer, Berlin-Göttingen-Heidelberg.

-(1953). Verh. dtsch. Ges. KreisForsch., 19, 52.

Spielmeyer, W. (1927). Z. ges. Neurol. Psychiat., 109, 501. 